\title{
Analysis of negative differential conductance in a two-island Coulomb blockade system by a polytope approximation in phase space
}

\author{
Gareth J. Evans \\ Microelectronics Research Centre, Cavendish Laboratory, Madingley Road, Cambridge CB3 OHE, \\ United Kingdom \\ H. Mizutaa) \\ Hitachi Cambridge Laboratory, Cavendish Laboratory, Madingley Road, Cambridge CB3 OHE, \\ United Kingdom and CREST, Japan Science and Technology (JST), Shibuya TK Bldg., 3-13-11 Shibuya, \\ Shibuya-ku, Tokyo 150-0002, Japan
}

(Received 14 March 2002; accepted for publication 25 June 2002)

\begin{abstract}
A two-island single-electron tunneling system is presented that exhibits negative differential conductance (NDC) based on Coulomb blockade. The NDC mechanism is explained by introducing a simple analysis method, the polytope approximation. A condition for NDC to occur is analyzed fully by using the polytope approximation. (C) 2002 American Institute of Physics.
\end{abstract}

[DOI: $10.1063 / 1.1500786]$

\section{INTRODUCTION}

Single-electron (SE) effects based on the Coulomb blockade (CB) of charge allows the manipulation of current on a per-electron basis. This has stimulated research into the possibility of using such effects to represent one bit of information by a small number of electrons and is hoped to lead eventually to a new generation of digital logic technology. The authors in Refs. 1 and 2 propose interesting devices based on negative differential conductance (NDC).

This article consists of two strands; the first demonstrates, through numerical simulation, a simple two-island SE device that exhibits NDC. NDC is usually associated with quantum levels but, due to SE effects, NDC need does not require energy quantization.

In the second strand, a simple analysis tool, called the polytope approximation, is proposed that neatly describes the reason for NDC and is used to derive a condition for NDC to occur. The polytope approximation is used to predict NDC valley positions and currents, both of which compare favorably with simulations.

Previously, Nakashima and Uozumi have demonstrated NDC in a linear array of seven ${ }^{3}$ and a nine $e^{4}$ islands in the context of a zig-zag conduction path through a granular system between two electrodes. The results were explained in terms of the competition between the forward rates of injection of charge into the array with increasing bias and the reduction of the tunneling rate across a junction that is against the electric field set up by the source-drain bias and so this rate reduces with increasing bias.

Shin et al. ${ }^{5,6}$ demonstrate a different form of NDC in closed rings of islands (the simplest being four islands). In this system, stable configurations of electrons can exist on the islands that form a "crystallike" structure and only when the bias has increased sufficiently to "melt" the crystal can current flow until a different stable configuration occurs. At

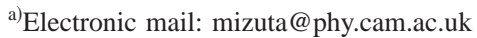

$T=0 \mathrm{~K}$, this leads to multiple zero-current regions as the source-drain bias increases, while at finite temperatures, it exhibits finite current and NDC.

Heij et al. ${ }^{7}$ take a simpler approach and attach a SE box to the gate of a SE transistor (SET) and demonstrate that the system will exhibit NDC for a range of conditions. The electrons in the box act as an additional gate bias to the SET and by biasing the SET and box in the appropriate range, the phase of the SET oscillation can be modified to produce NDC when an extra electron tunnels into/out of the box. A similar system is analyzed by Shin et $^{\mathrm{al}}{ }^{8}$ in the context of coupled SETs though the possibility of it generating NDC is not discussed.

SE devices show an extreme sensitivity to stray (uncontrolled) charge in the surrounding environment (the offsetcharge problem), but we will assume that a suitable fabrication process lets us control this. Under these circumstances, NDC devices may be a useful component of future SE logic systems.

\section{TWO-ISLAND COULOMB BLOCKADE SYSTEM}

The circuit that will be analyzed is shown in Fig. 1 and is similar to $\mathrm{Heij}^{7}$ et al.'s system in that it has two islands, but, like Nakashima and Uozumi's ${ }^{3,4}$ device, is arranged linearly such that electrons have to pass through both islands. We assume that islands are metallic and that their tunnel resistances and capacitances are independent of bias.

Despite its apparent simplicity, the system exhibits a variety of complex behaviors as the capacitances and the tunnel resistances are varied. However, this article only examines a simple case where all the tunnel resistances are set to $10^{6} \Omega$. The system exhibits NDC over a wide range of circuit parameters.

This circuit is the most general linear two-island circuit. Capacitances in parallel to the tunnel junctions can be merged together; capacitances to external gates are merged into the island-to-drain capacitance and the offset charge on the island changed appropriately. 


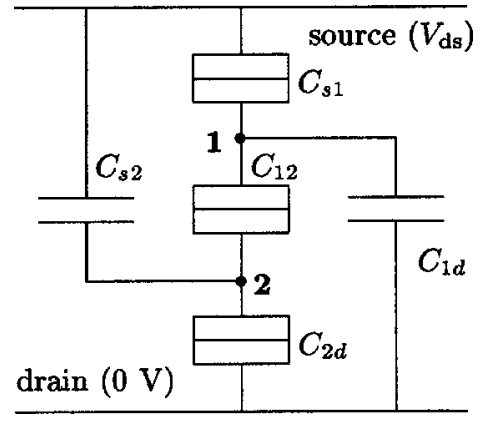

FIG. 1. Two-island CB system.

As an example of a system that demonstrates NDC, we set $C_{s 1}=C_{12}=C_{d 2}=0.1 \mathrm{aF}, C_{s 2}=6 \mathrm{aF}$, and $C_{d 1}=1 \mathrm{aF}$; the offset charge on islands 1 and 2 are denoted by $q_{1}$ and $q_{2}$ which are assumed to be in units of $e$. The results are generated using a Master equation simulator ignoring cotunneling. ${ }^{9}$ Later, a condition for NDC will be derived.

In this situation, $V_{\mathrm{ds}}$ acts as a gate on the second island-producing oscillations in its transfer characteristics, and hence NDC. Eventually, the bias over the second transistor is sufficient high that the gate looses control.

The current as a function of $V_{\mathrm{ds}}$ and of $q_{1}$ is shown in Fig. 2. $q_{2}$ has been set as -0.5 and, qualitatively, the only change in the characteristics as $q_{2}$ varies is that the phase of the oscillation changes. Clear NDC can be seen for a small range of the values of $q_{1}$; this demonstrates the extreme sensitivity of the characteristic on the offset charge. A change of only a small fraction of an electron charge can change the NDC current by many orders of magnitude or move the system to a non-NDC region.

Figure 7 shows the $I_{\mathrm{ds}}-V_{\mathrm{ds}}$ characteristics at temperatures between 8.2 to $1.2 \mathrm{~K}$ in $1 \mathrm{~K}$ steps. The NDC regions exhibit peak-to-valley ratios of up to $10^{10}$ at $4.2 \mathrm{~K}$. The peak heights for higher biases tend to be only weakly dependent on temperature while the valley currents are much more strongly dependent. This view is shown even more clearly for the different offset charge configuration $(-0.4,-0.5)$ which is shown in Fig. 8. Higher temperatures broaden the peaks, gradually washing them out.

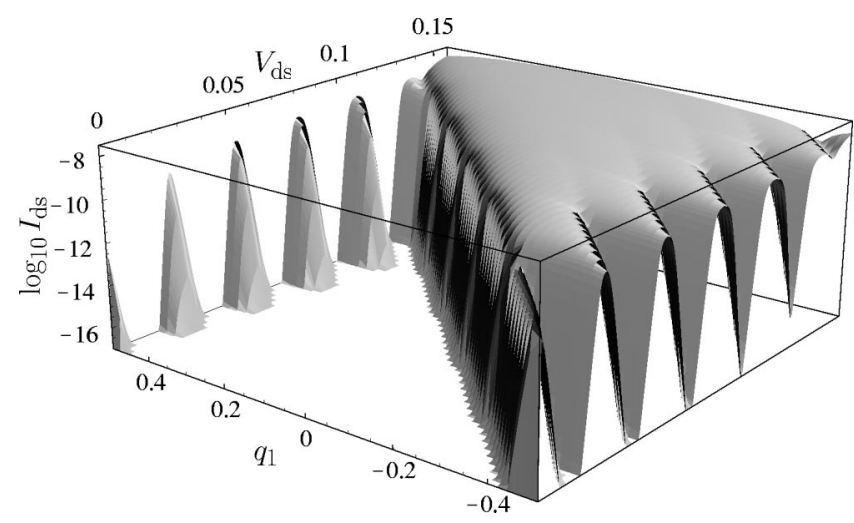

FIG. 2. $\log _{10} I\left(q_{1}, V_{\mathrm{ds}}\right)$ with $q_{2}=-0.5$-clearly there are values of $q_{1}$ where a small change produces a drastic change in the current and the existence of NDC.
Comparing Figs. 7 and 8, it is clear that the offset charge change of only $\Delta q_{1}=0.15$ (remembering that the characteristics are periodic in $q_{1}$ and $q_{2}$ ) has produced peaks that are a couple of orders of magnitude higher, while also degrading the quality of the peaks.

As a consequence, any logic device relying on the order of magnitude of the current would have to control $q_{1}$ to $\ll 0.15$, while the phase of the peaks is controlled by $q_{2}$ and a change of 0.5 produces a phase shift of $\pi$.

\section{ANALYSIS BASED ON THE POLYTOPE APPROXIMATION}

In this section, $\widetilde{\boldsymbol{q}}$, the phase space for the system, is introduced. A simple non-NDC example is used to motivate an approximation to the full Master equation solution to the current. Using this polytope $e^{10}$ approximation, the explanation of NDC is simply described. The condition for NDC to occur is then derived from this interpretation and the system is related to the device of Nakashima et al. ${ }^{3,4}$

The transition rate in Orthodox Theory ${ }^{11}$ for a hole tunneling through a tunnel resistance $R_{k l}$ from island $k$ to island $\ell$ is,

$$
\Gamma_{k l}=\frac{1}{e^{2} R_{k l}} \frac{-\Delta E_{k l}}{1-\exp \left(\Delta E_{k l} / k T\right)},
$$

where the change in free energy $\Delta E_{k l}$ can be expressed as,

$$
\Delta E_{k l}=\frac{e^{2}}{2} \boldsymbol{\epsilon}_{k l}^{T} \mathrm{C}^{-1} \boldsymbol{\epsilon}_{k l}+e^{2} \boldsymbol{\epsilon}_{k l}^{T} \mathrm{C}^{-1} \widetilde{\boldsymbol{q}}-e V_{k} \xi_{k}+e V_{\ell} \xi_{\ell}
$$

and each island $i$ is represented by the $i$ th element of the vectors. $\mathrm{C}$ is the (symmetric) capacitance matrix and $C_{i j}$ is the negative sum of the capacitances joining islands $i$ and $j$ while $C_{i i}$ is the positive sum of all the capacitances connected to island $i .\left(\boldsymbol{\epsilon}_{k l}\right)_{i}=-\delta_{i k}+\delta_{i \ell}$ and $\delta_{i j}$ is zero if $j$ is a bias. $\xi_{i}$ is unity if $i$ represents a bias, otherwise is zero. The operator $^{T}$ is the transpose of the vector.

The dynamics of the system are Markovian and for a fixed $V_{\mathrm{ds}}$ the state of the system is fully characterized by $(\widetilde{\boldsymbol{q}})_{i}=q_{i}+\Sigma C_{i^{\prime} i} V_{i^{\prime}} / e$, where $q_{i}=n_{i}+q_{i}^{0} / e$, the number of holes on island $i$ is $n_{i}$, the fractional offset charge induced on the island is $q_{i}^{0}$, and the sum $i^{\prime}$ runs over the capacitances/tunnel resistances connecting $i$ to bias/gate $i^{\prime}$ at potential $V_{i^{\prime}}$.

For the two-island system with the drain grounded, then,

$$
\mathrm{C}=\left(\begin{array}{cc}
C_{1 \Sigma} & -C_{12} \\
-C_{12} & C_{2 \Sigma}
\end{array}\right), \quad \widetilde{\boldsymbol{q}}=\left(\begin{array}{l}
q_{1}+C_{s 1} V_{\mathrm{ds}} / e \\
q_{2}+C_{s 2} V_{\mathrm{ds}} / e
\end{array}\right),
$$

where $C_{1 \Sigma}=C_{s 1}+C_{12}+C_{d 1}$, etc. Clearly the point describing the system $(\widetilde{\boldsymbol{q}})$ moves at a velocity of $\left(C_{s 1}, C_{s 2}\right)^{T} / e$ from a start point $\left(q_{1}, q_{2}\right)^{T}$.

The set of $\boldsymbol{\epsilon}_{k \ell}$ needed to move a hole from the source $(s)$ to the drain $(d)$ are $s \rightarrow 1: \boldsymbol{\epsilon}_{s 1}=(1,0)^{T}, \quad 1 \rightarrow 2: \boldsymbol{\epsilon}_{12}$ $=(-1,1)^{T}, 2 \rightarrow d: \boldsymbol{\epsilon}_{2 d}=(0,-1)^{T}$. If a transition $k \rightarrow \ell$ is taken then $\widetilde{\boldsymbol{q}} \rightarrow \widetilde{\boldsymbol{q}}+\boldsymbol{\epsilon}_{k \ell}$ and so a lattice of states is formed in $\widetilde{\boldsymbol{q}}$ space. The current may then be calculated by a Master equation connecting these lattice states using the formulas (1) and (2). A sequence of current-producing transitions make the system hop round a loop in the lattice. 


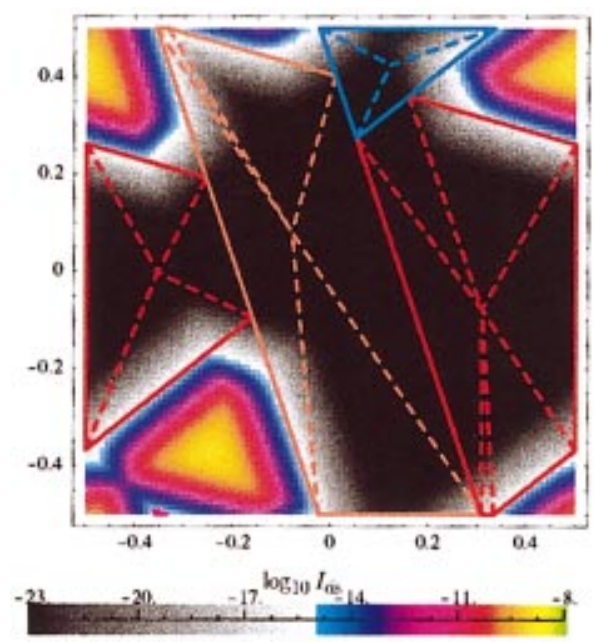

FIG. 3. (Color) Structure: $C_{s 1}=C_{12}=C_{d 2}=1 \mathrm{aF}, C_{d 1}=1.5 \mathrm{aF}$ and $V_{\mathrm{ds}}$ $=0.01 \mathrm{~V}$. The $\log _{10}$ of the current is plotted using the scale shown as a function of $\widetilde{\boldsymbol{q}}$. Note the periodicity of the characteristic and that surfaces of constant current are reasonably well described by straight line sections. The solid boundaries are those of the polytope approximation, the internal parts of the polytope are indicated by dashed lines.

For far-time dc characteristics, the initial lattice point that the system starts at is irrelevant as it will diffuse through the lattice and so the current is periodic in $\widetilde{\boldsymbol{q}}$ with the lattice spacing (unity). The polytope approximation assumes that this diffusion process takes the system to a set of loops involving a single state and that the transition rate out of this state dominates the current flow produced by any of the loops going through it. The dynamics of SE systems act such that if a particular transition $\boldsymbol{\epsilon}_{k \ell}$ from a state $\widetilde{\boldsymbol{q}}$ is taken then the transition rate for it being taken again from the new state $\left(\widetilde{\boldsymbol{q}}+\boldsymbol{\epsilon}_{k \ell}\right)$ is less $\left(\Gamma_{k \ell}[\widetilde{\boldsymbol{q}}]>\Gamma_{k \ell}\left[\widetilde{\boldsymbol{q}}+\boldsymbol{\epsilon}_{k \ell}\right]\right)$. Hence, the dynamics do indeed tend to confine the system to a small part of phase space.

A region of sub- $I_{\text {th }}$ current is constructed geometrically by calculating the region $\mathcal{Q}$ where all transition rates are less than $I_{\text {th }} / e$. Each point in $\widetilde{\boldsymbol{q}}$ space may now be classified as sub- $I_{\text {th }}$ if one (or more) of the points of the lattice that it is part of lies within $\mathcal{Q}$. In this way, a set of regions of sub- $I_{\text {th }}$ current is constructed and a lattice formed by translations of $\mathcal{Q}$. As the system is periodic, only a single $n$ cube of the space need be considered and this will be taken to be that centered at the origin and shall be denoted as $\mathcal{Q}_{0}$.

Figure 3 shows $\log _{10}\left[I_{\mathrm{ds}}(\widetilde{\boldsymbol{q}})\right]$ for a more capacitively homogeneous system. Surfaces of constant current are reasonably well approximated by polytopes, though there are neck points where the overall current is lower; the necks appear where two or more polytopes are in close proximity. The polytopes for 1 fA current are drawn; the original polytopes overlapped so a set of nonoverlapping polytopes were generated that covered the same area, these are drawn with solid boundaries and their interiors are marked by the dashed lines.

The polytope approximation, despite being so simple, tends to be conservative in which regions of phase space it assigns as sub- $I_{\text {th }}$, in that in all of the examples that the authors have studied, the true sub- $I_{\text {th }}$ region is a superset of the region that the polytope approximation provides. Strongly inhomogeneous tunnel resistances tend to lead to extra polytopelike regions that need further corrections to describe them. It is more difficult to test the validity of the polytope approximation in higher dimensions (more than two islands) but the terminology used in this paper is that for the general $n$-dimensional system in anticipation for such an extension.

A consequence of the polytope approximation is that, for a particular $V_{\mathrm{ds}}$, the volume of $\mathcal{Q}_{0}$ (in dimensionless units) is at least the probability that a device with a random offset charge configuration (uniformly distributed in the range -0.5 to 0.5 ) will have a threshold voltage below $V_{\mathrm{ds}} \cdot{ }^{12,13}$

\section{GEOMETRICAL EXPLANATION FOR NEGATIVE DIFFERENTIAL CONDUCTANCE IN THE TWO-ISLAND SYSTEM}

The phase space diagram of the two-island system for various values of $V_{\mathrm{ds}}$ is shown in Fig. 4. Each line of three diagrams are evenly spaced in $V_{\mathrm{ds}}$, there is a large jump in $V_{\mathrm{ds}}$ from one row to the other.

The polytope for this system is long and thin in the $q_{2}$ direction and wraps round many times. The polytopes drawn on the diagrams are for $I_{\mathrm{th}}=1 \mathrm{fA}$, the shading is indicative of the $\log _{10}\left(I_{\mathrm{ds}}\right)$ and the range of each diagram is from -0.5 to +0.5 in both directions. The system point starts at $(0.4$, $-0.5)$ and moves along the thick green line. Thus, in the top left-hand side diagram, the system is in a sub- $I_{\text {th }}$ region, the next in an above- $I_{\text {th }}$ region and the next again in a sub- $I_{\text {th }}$ region-thus, the system has gone through one current peak. We note that the trajectory of the system has wrapped round due to the periodic dependence on $\widetilde{\boldsymbol{q}}$.

The system travels very quickly in the $q_{2}$ direction, lapping the polytope many times while at the same time, the left-hand side edge of the polytope gradually moves to the right-hand side. The bottom set of diagrams show that polytope decreases in size until eventually it will disappear.

The non-NDC part of Fig. 2 can also be explained. Take for example the center point $(0,0)$. As $V_{\mathrm{ds}}$ increases, then it moves quickly in the $q_{2}$ direction, lapping many times, however, as the polytope at that point is all sub- $I_{\text {th }}$, then, the current is sub- $I_{\text {th }}$ until suddenly the left-hand side boundary of the polytope runs past it and it has a significant current.

\section{GEOMETRICAL CONDITION FOR NEGATIVE DIFFERENTIAL CONDUCTANCE}

We define that a device exhibiting NDC has a current that falls from above $I_{\text {th }}$ to below $I_{\text {th }}$ as $V_{\mathrm{ds}}$ increases i.e., $\ni V:\left[d I(V) / d V_{\mathrm{ds}}<0\right.$ and $\left.I(V)=I_{\mathrm{th}}\right]$. This is a more specific definition of NDC than usually used ( $\left.\ni V: d I(V) / d V_{\mathrm{ds}}<0\right)$.

A necessary condition for NDC is that the system can head from a point outside the polytope (with $I>I_{\text {th }}$ ) into the polytope (where $I<I_{\text {th }}$ ). Thus, the system must move faster toward a polytope boundary faster than the boundary recedes. This can be done for each face and can be used to construct a region of phase space $(\widetilde{\boldsymbol{q}})$ that can hit the face before it completely disappears - thus providing a criterion under which at least one dip of at least $I_{\text {th }}$ can occur. 

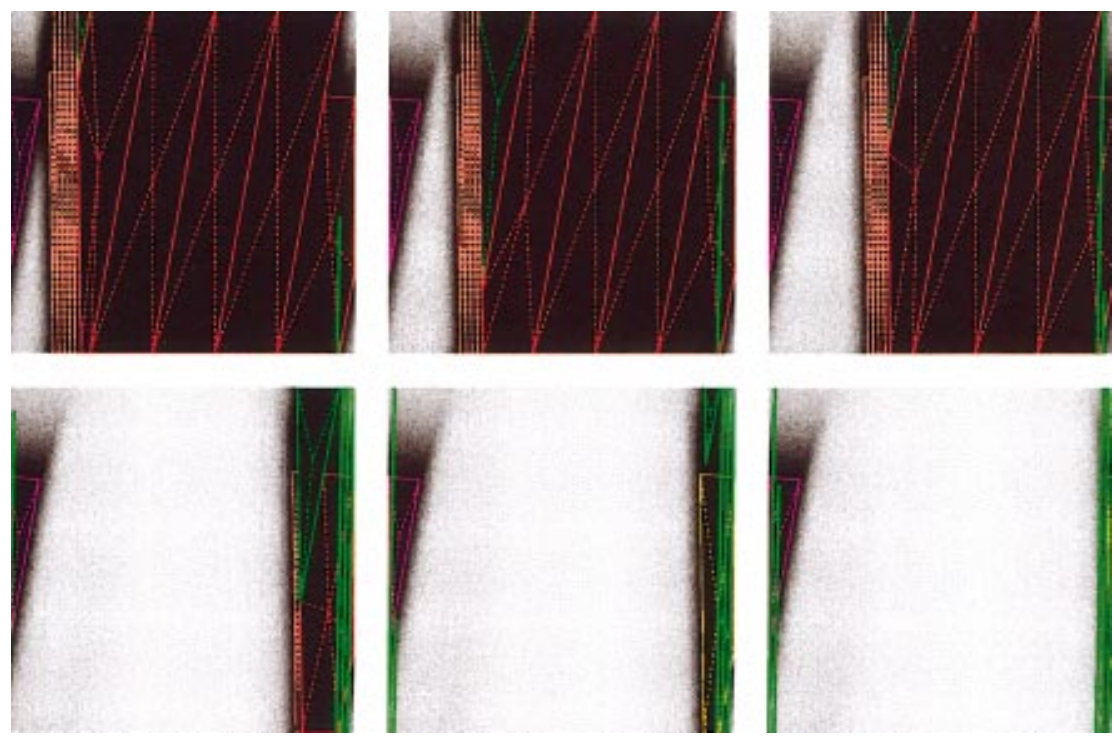

FIG. 4. (Color) Phase space diagrams for an NDC system. The polytopes have solid boundaries and their interiors are marked by dashed lines. $V_{\mathrm{ds}}$ in the top row of diagrams are 10.5, 21.0, and $31.5 \mathrm{mV}$ (left- to right-hand side), while $V_{\mathrm{ds}}$ for the bottom rows are 105, 115 , and $126 \mathrm{mV}$. The system point starts at $(0.4,-0.5)$ and moves along the thick green line (that is almost vertical). As $V_{\mathrm{ds}}$ increases from one diagram to the next, the line moves out of a subthreshold (1 fA) region and back again. The bottom set of diagrams shows the system shortly before the polytope disappears and all points have above threshold current.
The phase-space diagram for a voltage $V_{\mathrm{ds}}$ is drawn in Fig. 5, the sub- $I_{\text {th }}$ region is the triangle $A B C$, the shaded triangle is one of the images of $A B C$ due to the periodicity in $\widetilde{\boldsymbol{q}}$ space. The transition $s \rightarrow 1$ corresponds to the edge $B C$ and this is the only edge that moves, and its velocity is $\boldsymbol{v}_{s 1}$. The edge $A B$ corresponds to the interisland transition $1 \rightarrow 2$, while $C A$ is the transition $2 \rightarrow d$. The normal to the face $A B$ is $\boldsymbol{n}_{12}$. The system is described by the point $\widetilde{\boldsymbol{q}}$, indicated by the circle, and travels at velocity $\boldsymbol{v}_{q}=\left(C_{s 1}, C_{s 2}\right)^{T} / e$.

The triangle $A B C$ gradually shrinks until it disappears to the point $A$ at a voltage $V_{\mathrm{ds}}^{\max }$. All points in the triangle $A X B$ will have flowed through $A B$ by this time and so will have experienced at least one section of NDC between $V_{\mathrm{ds}}$ and $V_{\mathrm{ds}}^{\max }$ as they move from a point outside the triangle (where

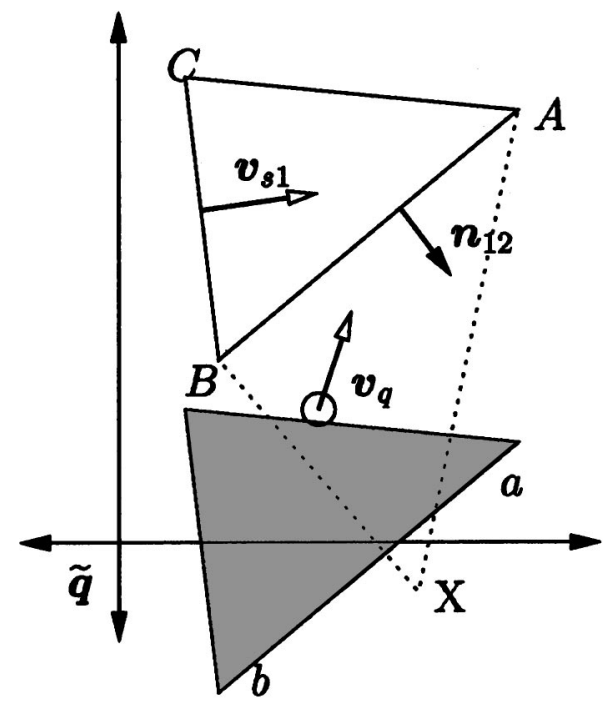

FIG. 5. Phase-space diagram: the interior of the polytope $A B C$ is a region of sub- $I_{\text {th }}$ current. $B C$ moves to the right-hand side with increasing $V_{\mathrm{ds}}$ at a velocity $\boldsymbol{v}_{s 1}$, the lines that the other edges are part of are not dependent on $V_{\mathrm{ds}}$. The shaded triangle is one of the periodic images of $A B C$. The outward normal to the edge $A B$ is $\boldsymbol{n}_{12}$. A particular system, indicated by the circle, moves at a velocity $\boldsymbol{v}_{q}$. A system in the region $A X B$ will move through $A B$ before the polytope disappears and thus will exhibit NDC.
$\left.I>I_{\text {th }}\right)$ to inside $\left(I<I_{\text {th }}\right)$. Those points that also cross through $a b$ will have two NDC sections with $I>I_{\text {th }}$.

The outward pointing normal to the region for a transition $\boldsymbol{\epsilon}_{k \ell}$ is $-\mathrm{C}^{-1} \boldsymbol{\epsilon}_{k \ell}$, and so a necessary condition for the system to be able to go through face $A B$ is that $\boldsymbol{v}_{q}$ is directed oppositely to the outward normal to $A B\left(\boldsymbol{n}_{12}\right)$,

$$
\begin{aligned}
& \boldsymbol{v}_{q} \cdot \boldsymbol{n}_{12}<0 \\
& \left(C_{s 1}, C_{s 2}\right)\left(\begin{array}{l}
C_{2 \Sigma}-C_{12} \\
C_{12}-C_{1 \Sigma}
\end{array}\right)<0 \\
& C_{s 2}\left(C_{s 1}+C_{d 1}\right)>C_{s 1}\left(C_{s 2}+C_{d 2}\right) \\
& \frac{C_{d 1}}{C_{s 1}}>\frac{C_{d 2}}{C_{s 2}} .
\end{aligned}
$$

Similarly, the condition for the system to be able to go through $B C$ is,

$$
\begin{aligned}
& \boldsymbol{v}_{q} \cdot\left(-\boldsymbol{n}_{s 1}\right)-\left|\boldsymbol{v}_{s 1}\right|>0 \\
& \left(C_{s 1}, C_{s 2}\right) \cdot\left(\begin{array}{c}
C_{2 \Sigma} \\
C_{12}
\end{array}\right)-\left(C_{1 \Sigma} C_{2 \Sigma}-C_{12}^{2}\right)>0 \\
& C_{s 1} C_{2 \Sigma}+C_{s 2} C_{12}-C_{1 \Sigma} C_{2 \Sigma}+C_{12}^{2}>0 \\
& -C_{d 1} C_{2 \Sigma}-C_{12} C_{d 2}>0,
\end{aligned}
$$

which can never be satisfied.

Doing the same for edge $C A$,

$$
\begin{aligned}
& \boldsymbol{v}_{q} \cdot \boldsymbol{n}_{2 d}<0 \\
& \left(C_{s 1}, C_{s 2}\right) \cdot\left(\begin{array}{c}
C_{12} \\
C_{1 \Sigma}
\end{array}\right)<0 \\
& C_{s 1} C_{12}+C_{s 2} C_{1 \Sigma}<0,
\end{aligned}
$$

which again can never happen (geometrically, this is because the outward normal and the system velocity are both lie in the same quadrant).

Thus, the system can only travel fast enough toward the face $A B$ and to do this it must obey Eq. (3). The physical interpretation of this is that the transition rate corresponding 


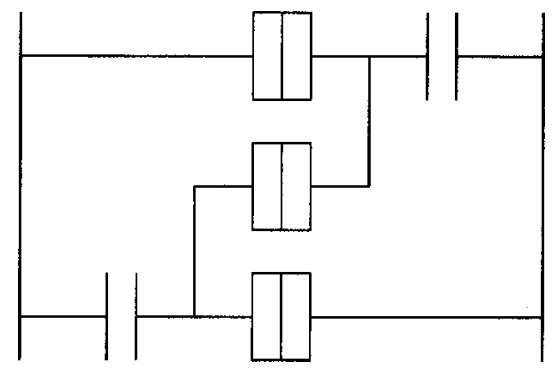

FIG. 6. The circuit remapped to a zig-zag path.

to the interisland transition $(1 \rightarrow 2)$ decreases with increasing $V_{\mathrm{ds}}$. The condition (3) implies that Fig. 1 can be redrawn more suggestively as Fig. 6 which now is reminiscent of Nakashima and Uozumi's, ${ }^{3,4}$ configuration-indeed, this is the simplest zig-zag pattern possible. Island one couples more strongly to the drain and island two to the source, this means that the potential drop across the central tunnel resistance is in an energetically unfavorable direction for holes to tunnel from island one to island two en route from source to drain.

The condition (3) is a necessary condition under the polytope approximation even in the case when the polytopes from adjacent lattice points overlap because the resultant above- $I_{\text {th }}$ region is bounded by line segments that have the same normals and velocities as the polytopes making it up $\left\{\boldsymbol{n}_{s 1}, \boldsymbol{n}_{12}, \boldsymbol{n}_{2 d}\right\}$. As a consequence, the condition required is identical.

Relaxing the definition for NDC to the most general case ( $\ni V: d I(V) / d V_{\mathrm{ds}}<0$ ) still delivers the same condition as it is independent of the choice of $I_{\text {th }}$.

As an aside, the parameters can be chosen such that the system point moves parallel to the edge $A B$ of the polytope and thus the current remains constant while this is the dominant edge. Trivially, the condition to produce this constant current step is obtained by setting the inequality of Eq. (3) to equality.

The polytope method implies that the prediction of the minimum current is an easily accessible quantity. In general, it is difficult to write analytic formulae for these currents due to the periodicity of the space. However, in our specific example, then the minimum current should be when the transition rate for processes $\boldsymbol{\epsilon}_{2 d}$ and $\boldsymbol{\epsilon}_{12}$ are identical.

If both tunnel resistances are identical then, this is equivalent to saying their transition energies are identical:

$$
\begin{aligned}
& \boldsymbol{\epsilon}_{2 d}^{T} \mathrm{C}^{-1} \widetilde{\boldsymbol{q}}+\frac{1}{2} \boldsymbol{\epsilon}_{2 d}^{T} \mathrm{C}^{-1} \boldsymbol{\epsilon}_{2 d}=\boldsymbol{\epsilon}_{12}^{T} \mathrm{C}^{-1} \tilde{\boldsymbol{q}}+\frac{1}{2} \boldsymbol{\epsilon}_{12}^{T} \mathrm{C}^{-1} \boldsymbol{\epsilon}_{12} \\
& \left(\boldsymbol{\epsilon}_{2 d}^{T}-\boldsymbol{\epsilon}_{12}^{T}\right) \mathrm{C}^{-1} \widetilde{\boldsymbol{q}}=-\frac{1}{2}\left[\boldsymbol{\epsilon}_{2 d}^{T} \mathrm{C}^{-1} \boldsymbol{\epsilon}_{2 d}+\boldsymbol{\epsilon}_{12}^{T} \mathrm{C}^{-1} \boldsymbol{\epsilon}_{12}\right] \\
& \frac{e^{2}}{|\mathrm{C}|}\left(\begin{array}{c}
2 C_{12}-C_{2 \Sigma} \\
-C_{12}+2 C_{1 \Sigma}
\end{array}\right) \cdot \widetilde{\boldsymbol{q}}=\frac{e^{2}\left(C_{2 \Sigma}-C_{12}\right)}{2|\mathrm{C}|} \\
& \left(\begin{array}{c}
2 C_{12}-C_{2 \Sigma} \\
-C_{12}+2 C_{1 \Sigma}
\end{array}\right) \cdot \widetilde{\boldsymbol{q}}=\frac{C_{2 \Sigma}-C_{12}}{2},
\end{aligned}
$$

this line intersects the system velocity point $\widetilde{\boldsymbol{q}}=\left(q_{1}, q_{2}\right)$ $+\left(C_{s 1}, C_{s 2}\right) V_{\mathrm{ds}} / e$ when,

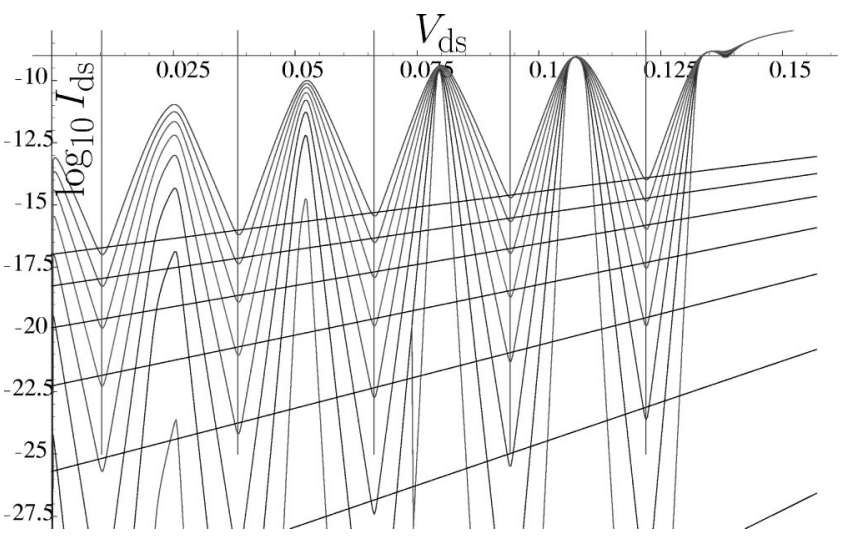

FIG. 7. $(0.4,-0.5): \log _{10} I_{\mathrm{ds}}\left(V_{\mathrm{ds}}\right)$ characteristics at temperatures $8.2 \mathrm{~K}$ (top) to $1.2 \mathrm{~K}$ (bottom) in $1 \mathrm{~K}$ steps. Marked by vertical lines are the predicted positions of minimum currents and also are shown the predicted currents by the nonoscillating lines.

$V_{\mathrm{ds}}=e$

$$
\cdot \frac{\left(C_{2 \Sigma}-C_{12}\right) / 2-\left[q_{1}\left(2 C_{12}-C_{2 \Sigma}\right)+q_{2}\left(-C_{12}+2 C_{1 \Sigma}\right)\right]}{C_{s 1}\left(2 C_{12}-C_{2 \Sigma}\right)+C_{s 2}\left(-C_{12}+2 C_{1 \Sigma}\right)},
$$

and so the coefficient of $q_{2}$ is the period of the oscillations,

$$
\begin{aligned}
\Delta V_{\mathrm{ds}} & =e \cdot \frac{-C_{12}+2 C_{1 \Sigma}}{C_{s 1}\left(2 C_{12}-C_{2 \Sigma}\right)+C_{s 2}\left(-C_{12}+2 C_{1 \Sigma}\right)} \\
& =\frac{e}{C_{s 2}} \cdot \frac{1}{1-\frac{C_{s 1}}{C_{s 2}} \cdot \frac{C_{2 \Sigma}-2 C_{12}}{2 C_{1 \Sigma}-C_{12}}},
\end{aligned}
$$

for our example system this gives $0.0279 \mathrm{~V}$ which is within error margins of the numerical simulation. The second factor in this term increases the period by $\approx 4.5 \%$ compared to $e / C_{s 2}$.

The $V_{\mathrm{ds}}$ positions of the valleys can also be found from Eq. (7) though care is needed to cope with the periodicity of the space. These are shown by the vertical red lines in Figs. 7 and 8. From their intersection points, the current can also be estimated and these are draw using black lines on the figures.

The agreement of valley positions is excellent, however, the current predictions are too high by about a factor two for Fig. 7 and, for some cases in Fig. 8, by over an order of magnitude. This poor agreement is due to the breakdown of the assumptions of the polytope approximation. In these cases, there are at least two loops of states and the lowest transition rate step may not be the limiting factor of the loop as reverse transition rates (holes hopping backwards) may limit the current.

\section{CONCLUSIONS}

This article has introduced the polytope approximation as a tool to analyze the behavior of SE systems. It has been used to demonstrate that a two-island SET system with no quantum energy levels can exhibit NDC due to CB. Furthermore, from a purely geometric point of view, a condition for 


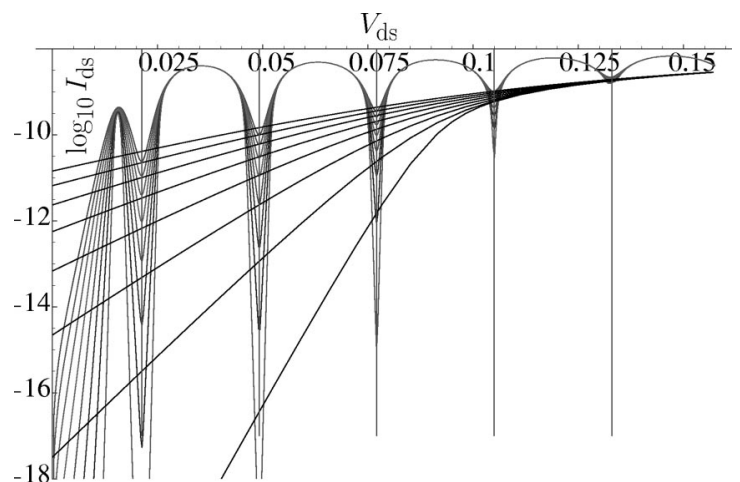

FIG. 8. $(-0.45,-0.5): \log _{10} I_{\mathrm{ds}}\left(V_{\mathrm{ds}}\right)$ characteristics at temperatures $8.2 \mathrm{~K}$ (top) to $1.2 \mathrm{~K}$ (bottom) in $1 \mathrm{~K}$ steps. Marked by vertical lines are the predicted positions of minimum currents and also are shown the predicted currents by the nonoscillating lines.

NDC has been derived by showing that the system must move through phase space at sufficient velocity that it can pass through one of the faces of the polytope. This leads to an algebraic condition for NDC.
${ }^{1}$ K. Maezawa, T. Akeyoshi, and T. Mizutani, IEEE Trans. Electron Devices 41, 148 (1994).

${ }^{2}$ H. J. Levy and T. C. McGill, IEEE Trans. Neural Netw. 4, 427 (1993).

${ }^{3}$ H. Nakashima and K. Uozumi, Jpn. J. Appl. Phys., Part 2 34, L1659 (1995).

${ }^{4}$ H. Nakashima and K. Uozumi, J. Vac. Sci. Technol. B 15, 1411 (1997).

${ }^{5}$ M. Shin, S. Lee, K. W. Park, and E. H. Lee, J. Appl. Phys. 84, 2974 (1998).

${ }^{6}$ M. Shin, S. Lee, K. W. Park, and E. H. Lee, Phys. Rev. Lett. 80, 5774 (1998).

${ }^{7}$ C. P. Heij, D. C. Dixon, P. Hadley, and J. E. Mooij, Appl. Phys. Lett. 74, 1042 (1999)

${ }^{8}$ M. Shin, S. Lee, and K. W. Park, Phys. Rev. B 62, 9951 (2000).

${ }^{9}$ M. Kirihara, K. Nakazato, and M. Wagner, Jpn. J. Appl. Phys., Part 1 38, 2028 (1999).

${ }^{10} \mathrm{~A}$ polytope is the mathematical name for a finite volume polyhedron. In general, $n$-dimensional terminology will be used in this article-thus a plane [an $(n-1)$-dimensional object] is a line in two dimensions, a volume is an area in two dimensions, etc.

${ }^{11}$ Single Charge Tunneling, edited by H. Grabert and M. H. Devoret (Plenum, New York, 1992).

${ }^{12}$ G. J. Evans, H. Mizuta, and H. Ahmed, Jpn. J. Appl. Phys., Part 1 84, 2974 (2001).

${ }^{13}$ G. J. Evans, Ph.D. thesis, University of Cambridge, 2002. 